\title{
Advanced In Situ I-V Measurements Used in the Study of Porous Structures Growth on Silicon
}

\author{
Amare Benor ${ }^{1,2}$ \\ ${ }^{1}$ Department of Physics, Bahir Dar University, P.O. Box 79, Bahir Dar, Ethiopia \\ ${ }^{2}$ Department of Physics, Addis Ababa University, P.O. Box 1176, Addis Ababa, Ethiopia \\ Correspondence should be addressed to Amare Benor; amarebenor@gmail.com
}

Received 14 April 2017; Revised 10 July 2017; Accepted 25 July 2017; Published 6 September 2017

Academic Editor: P. Davide Cozzoli

Copyright $\odot 2017$ Amare Benor. This is an open access article distributed under the Creative Commons Attribution License, which permits unrestricted use, distribution, and reproduction in any medium, provided the original work is properly cited.

\begin{abstract}
The rate of oxide formation during growth of pores structures on silicon was investigated by in situ $I-V$ measurements. The measurements were designed to get two $I-V$ curves in a short time (total time for the two measurements was 300 seconds) taking into account the gap (in $\mathrm{mA} / \mathrm{cm}^{2}$ ) for each corresponding voltage. The in situ $I-V$ measurements were made at different pore depth/time, at the electrolyte-pore tip interface, while etching takes place based on p-type Si. The results showed increasing, decreasing, and constant $I-V$ gap in time, for macropores, nanopores, and electropolishing regimes, respectively. This was related to the expected diffusion limitation of oxide forming $\left(\mathrm{H}_{2} \mathrm{O}\right)$ molecules reaching the electrolyte-pore tip and the anodizing current, while etching takes place. The method can be developed further and has the potential to be applied in other electrochemically etched porous semiconductor materials.
\end{abstract}

\section{Introduction}

A startling and groundbreaking insight into porous silicon appeared towards the middle of the last century [1]. Yet during that time, scientific details on the porous silicon were not recognized well and the issue was not tackled for some time after the initial studies [2]. Thereafter, in the late 1980s, the science of porous silicon has shown some advances, and the studies were made by altering electrochemical etching conditions [3]. In 1990, Lehmann and Föll also discovered that very regular arrays of macropores with extremely large aspect ratio could be obtained in $\mathrm{n}-\mathrm{Si} / \mathrm{HF}$ system, and they explained the phenomenon using the "Space Charge Region" model [4]. The discovery of porous silicon opened new possibilities and extended applications in three-dimensional semiconductor structures [2], sensors [5, 6], microelectromechanical system (MEMS) [7], photonic devices [8], and the like. In relation to such novel applications, the issue of porous silicon structures has also gained considerable attention in the last two decades. The studies expand the scope of our understanding on the possible electrochemical process, reaction kinetics on oxide formation, and electropolishing mechanisms at the siliconelectrolyte interface. Particular studies on the oxidization mechanisms at the silicon-electrolyte interface were also made in the past $[4,9]$. Some of the past studies have shown that the rate of oxidation is affected by electrolyte concentration, anodizing current density, and diffusion limitation of oxide forming $\left(\mathrm{H}_{2} \mathrm{O}\right)$ molecules $[4,6]$. Such studies have extended our understanding of the oxide forming mechanism for pore formations. However, the rate of oxide formation during pores formation is still something not clearly understood $[4,10]$. Thus, in this study, we used a new method hereafter referred to as "fast $I-V$ curve" to study the rate of oxide formation during pore formations and electropolishing regimes. Consequently, the effects of anodizing current, pore morphology, and pore type on the rate of oxide formation are crucial elements of this study.

The fast $I-V$ characterization method is designed to get two $I-V$ measurements within short time $(300 \mathrm{sec})$. Compared to the first measurement, the second $I-V$ measurement, one in forward and the other in reverse $I-V$ measurements, shows displacement of the current (in $\mathrm{mA} / \mathrm{cm}^{2}$ ), for each particular voltage values. The periodic fast $I-V$ measurements were considered six times for each experiment completed within 360 or $120 \mathrm{~min}$. The displacement of the current attributes to the oxide formation in the time range between 


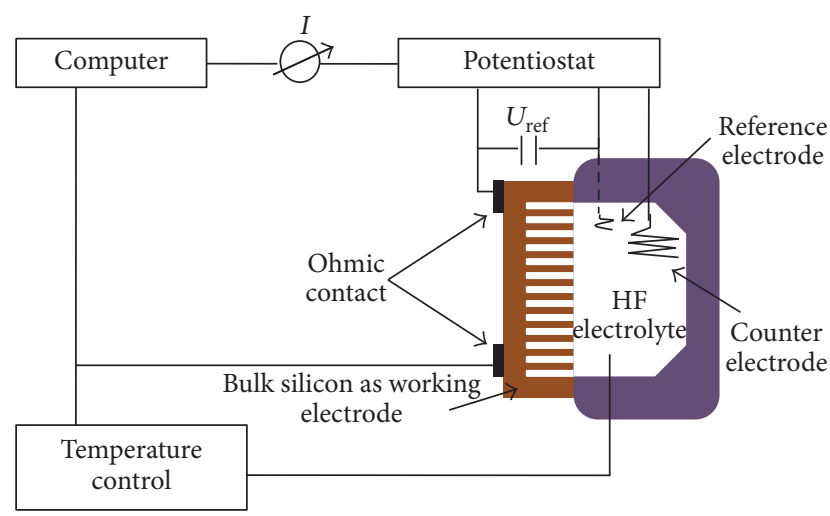

FIgURE 1: Schematic illustration of the etching cell.

the two measurements, where it is affected by electrochemical pore regimes, that is, macroporous, meso/nanoporous, and electropolishing. Besides, it is worth mentioning that the rate of oxidation also depends on diffusion rate of the oxide forming $\left(\mathrm{OH}^{-}\right)$molecules reaching at the pore tip-semiconductor interface, where the diffusion in turn depends on the morphology, type, and depth of the pores that change in time during etching $[2,11]$. The pore types, pore etching depth, and pore morphology were characterized by SEM (Scanning Electron Microscopy) after the multiple in situ $I-V$ measurements. Thereafter, based on the ex situ (SEM) and in situ ( $I-V$ gap) measurements, a comparative rate of oxide formation, during pore formation and electropolishing, was made as a function of time. In other words, rate of oxide formation is also studied indirectly as a function of pore depth in the porous regime. Thus, in this study, the new method is used to study the rate of oxide formation during porous and electropolishing regimes at different time for the p-type $\mathrm{Si}$, and the method can be extended to other porous semiconductor materials including n-type $\mathrm{Si}$ and other porous single crystalline semiconductors.

\section{Experimental Details}

The investigations were done on p-type (100) wafer with resistively of $10-20 \Omega \cdot \mathrm{cm}$. In order to get a good ohmic contact, the samples were dipped into $10 \% \mathrm{HF}$. After removing the oxide by scratching, a layer of In/Ga alloy was applied to the sample for contact. Consequently, the samples are introduced into the cell (see Figure 1) [12,13], and the experimental parameters including current density and etching time were computer controlled. The electrolyte contains $4 \% \mathrm{wt}$. HF without surfactant/tenside addition. The experiments were conducted at a constant temperature of $20^{\circ} \mathrm{C}$ and constant pumping rate $(100 \mathrm{rpm})$. The current density $(j)$ for a single experiment was constant during the experiment (except during the periodic fast $I-V$ measurement) but varied from experiment to experiment between $j=0.1 \mathrm{~mA} / \mathrm{cm}^{2}$ and $j=30 \mathrm{~mA} / \mathrm{cm}^{2}$ (voltage $-0.5-1.5 \mathrm{~V}$ ). The etching time was adjusted to $120 \mathrm{~min}$, and for some cases, where it was not possible to get pores with $120 \mathrm{~min}$, the time was increased to $360 \mathrm{~min}$ and the time effect was considered. However, unlike the macropores etched for $360 \mathrm{~min}$, both nanopores and electropolishing were achieved at the lower time and the in situ characterizations were performed for up to $120 \mathrm{~min}$. The time interval between the two

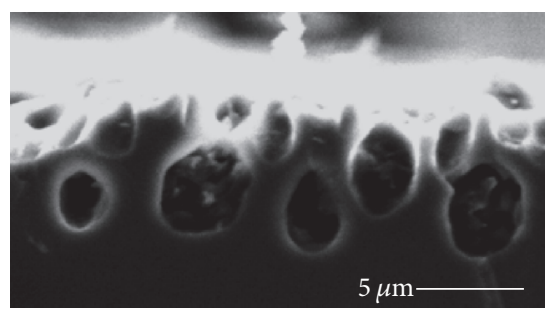

(a)

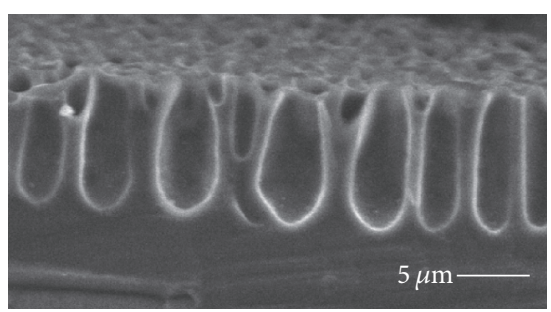

(b)

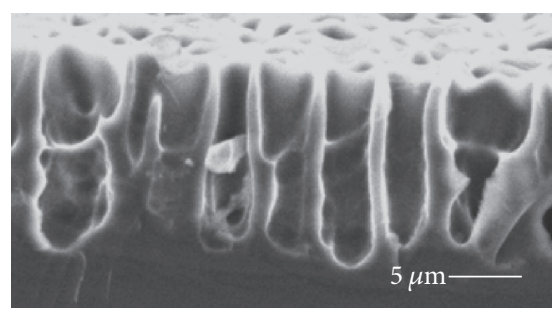

(c)

FIGURE 2: SEM images of the samples anodized at different current densities in $360 \mathrm{~min}$ at $0.5 \mathrm{~mA} / \mathrm{cm}^{2}$ (a), $1 \mathrm{~mA} / \mathrm{cm}^{2}$ (b), and $2 \mathrm{~mA} / \mathrm{cm}^{2}$ (c).

fast $I-V$ measurements was $300 \mathrm{sec}$ for all the experiments. Subsequently, the fast $I-V$ data, measured six times for single experiment/sample, were plotted and analyzed. At the end of the experiment, the sample was removed from the cell, rinsed in water, and broken in two parts for cross-section investigation of the porous morphology. The etched samples were investigated by means of SEM Philips XL30 microscope, that is, SEM as ex situ measurement.

\section{Results and Discussion}

Macropores were observed from 0.5 to $2 \mathrm{~mA} / \mathrm{cm}^{2}$ with etching time of $320 \mathrm{~min}$; however, it was not possible to get deep and straight macropores unlike the known cases using surfactant or tenside added electrolyte [14]. The nanopores start to grow at $3 \mathrm{~mA} / \mathrm{cm}^{2}$ and their percentage of surface coverage increases up to $15 \mathrm{~mA} / \mathrm{cm}^{2}$. Finally, a complete electropolishing was obtained at $25 \mathrm{~mA} / \mathrm{cm}^{2}$ (or higher). Thus, in the study, the three phases of the electrochemical etching of the porous silicon were observed: macropores, nanopores, and electropolishing.

3.1. Macropores. The etching times to get macropore structures were first tried at $120 \mathrm{~min}$, but no evident macropores were obtained. Thus, the time was extended to $360 \mathrm{~min}$ to get macropores. Figure 2 presents SEM images of the p-type 

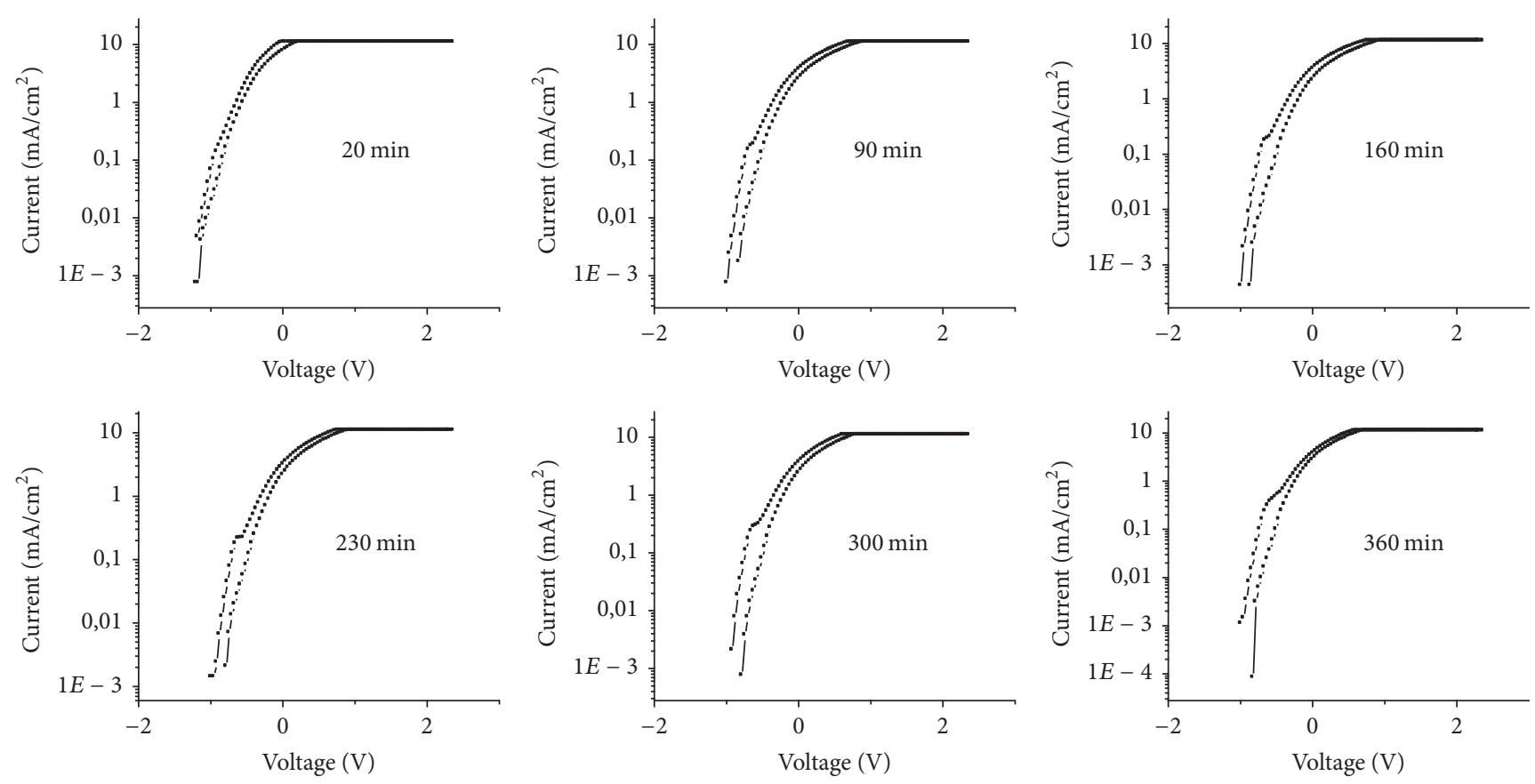

Figure 3: Typical in situ fast $I-V$ measurements (at 20, 90, 160, 230, 300, and 360 minutes) during the growth of macropores structures at $j$ $=1 \mathrm{~mA} / \mathrm{cm}^{2}$.

silicon etched at $1 \mathrm{~mA} / \mathrm{cm}^{2}$. When the pores are seen in crosssections, it is evident that pores are not perfectly straight and their opening is narrowed. Besides, the pores seem to show low aspect ratio and remain "shallow" in $360 \mathrm{~min}$ (e.g., $\sim 10 \mu \mathrm{m}$ at $1 \mathrm{~mA} / \mathrm{cm}^{2}$ ). Furthermore, when the pores are seen at different current densities, pores channel walls are not straight (see Figure 2). Instead, the pores structures are branched and spongy type, tumbler shape (narrowed opening and bulged base), and pores with cavity like structures at $0.5 \mathrm{~mA} / \mathrm{cm}^{2}, 1 \mathrm{~mA} / \mathrm{cm}^{2}$, and $2 \mathrm{~mA} / \mathrm{cm}^{2}$, respectively. Additionally, as shown in Figure 2, as the current density increases from 0.5 to $2 \mathrm{~mA} / \mathrm{cm}^{2}$, the pore growth rate increases. Such an enhancement of the pore growth rate, including pore depth and opening/diameter, by increasing the current is also consistent with other findings $[15,16]$. The anodizing current and the way pores are grown can also affect the rate of oxide formation and can be manifested in the fast $I-V$ gaps.

The in situ fast $I-V$ measurements for the macropores structures at $j=1 \mathrm{~mA} / \mathrm{cm}^{2}$ are presented in Figure 3. As seen in Figure 3, the fast $I-V$ gap between the forward and reverse $I-V$ tends to increase in time (from 20 to $360 \mathrm{~min}$ ). Yet, the increased gap seems to be noticeable in the first $90 \mathrm{~min}$ and thereafter the change/increase of the gap in time is smaller in extent. Similarly, the increase of the $I-V$ gap in time is also observed for the other macropores obtained at $j$ $=0.5 \mathrm{~mA} / \mathrm{cm}^{2}$ and $j=2 \mathrm{~mA} / \mathrm{cm}^{2}$. The increase in the fast $I-$ $V$ gap in time can be related to the morphology of the pores which change in time, that is, the morphology seems to create favorable mass transfer geometry in time. As a result, such an improved mass transfer, at the pore tip-semiconductor $(\mathrm{Si})$ interface, resulted in enhanced oxide thickness growth which in turn leads to increased $I-V$ gap. As can be observed from
Figure 1, the macropores are not straight and deep, instead, spongy like, having cavity or tumbler structure. The pores intersect with each other (mainly at $j=0.5 \mathrm{~mA} / \mathrm{cm}^{2}$ ) due to the fact that they increase their diameter as they grow into the substrate. The mass-transfer-controlled electrochemical etching is determined by diffusion of the oxide forming species and the diffusion depends on the geometry of pores $[11,17]$. However, the geometry of pores changes in time during etching. Such a change of pore morphology, to sponge or tumbler/cavity like structure, can increase diffusion of oxide forming species in time as pores grow down. This can be due to the dynamic pore geometry favoring/improving mass transfer in time or the effect of cross-connected pores (spongy) resulting in multidirectional mass transfer at the pore tip-Si interface enhancing oxide formation, for example, from two channels for two pore tip-Si interfaces to two channels for a single pore tip-Si interface. In other words, the amount of oxide forming species per pore tip area, at the active/etching site interface, within the pore seems to increase in time by multichannelled electrolyte path for spongy like structure. Similarly, in relation to the morphology of the pore for tumbler/cavity like structure, the amount of oxide forming species per pore tip area seems to increase as pores get deep. Thus, taking into account the fact that the fast $I-V$ gap was assumed to be related to the amount of oxide, one can expect that such structure increases the gap as the structure develops in time.

To get deep insight into the rate oxide formation, an extended experiment by varying the current densities $\left(0.5 \mathrm{~mA} / \mathrm{cm}^{2}, 1 \mathrm{~mA} / \mathrm{cm}^{2}\right.$, and $\left.2 \mathrm{~mA} / \mathrm{cm}^{2}\right)$ was made to study the $I-V$ gap at a specific or constant time, that is, $I-V$ gap at different current density. Figure 4 presents in situ fast $I-V$ 


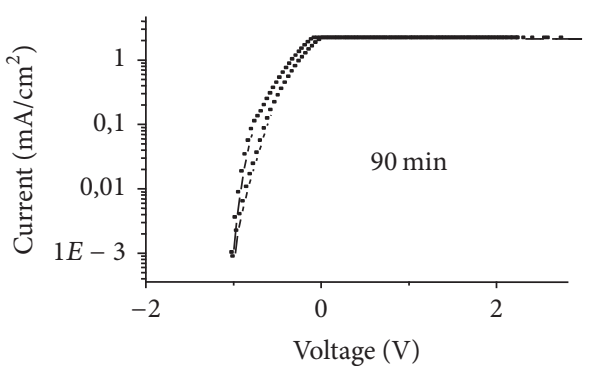

(a)

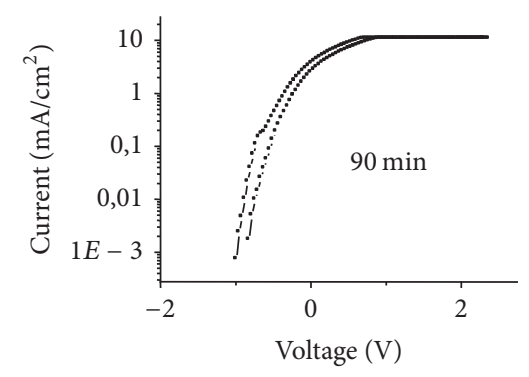

(b)

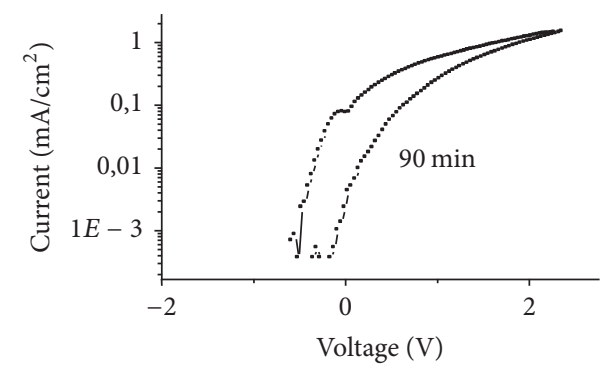

(c)

FIGURE 4: In situ fast $I-V$ measurements at 90 minutes during pore formation using anodizing current of $0.5 \mathrm{~mA} / \mathrm{cm}^{2}$ (a), $1 \mathrm{~mA} / \mathrm{cm}^{2}$ (b), and $2 \mathrm{~mA} / \mathrm{cm}^{2}$ (c).

measurements at $90 \mathrm{~min}$ for the corresponding macropores obtained at $0.5,1$, and $2 \mathrm{~mA} / \mathrm{cm}^{2}$. As shown in the figure, the $I-V$ gap visibly expands as the current density increases from 0.5 to $2 \mathrm{~mA} / \mathrm{cm}^{2}$. Similarly an increase of the fast $I-V$ gap by increasing the current density was also observed at each case, that is, 20, 160, 230, 300, and 360 minutes. Such a trend seems to indicate that the rise of current density, within the macropores regime, enhances oxide formation once the etching process starts. This is consistent to previous findings $[15,16]$. Here, the diffusion limitation of the oxide forming species can possibly vary in the three cases as there are differences in pores morphologies (see Figure 2). However, here, the significant increase in the $I-V$ gap at $90 \mathrm{~min}$ for the three cases (Figure 4) with the increase of the anodizing current tends to indicate that the anodizing current can have a more dominant role than the pore morphology in the oxide formation.

3.2. Nanopores. The electrochemical process at $3 \mathrm{~mA} / \mathrm{cm}^{2}$ did not show pore etching and seems to be at a point where growth of macropores terminates. Figure 5 presents the major etching process after the macrospores regime, that is, nanopores and electropolishing regimes. The macropores are easily seen by means of a microscope. This is not the case of nanopores, so an ultraviolet (UV) lamp illumination, UV excited photoluminescence, was used in order to prove the existence of nanopores [18-20]. Here, it is important to note difficulty in differentiating between mesopores and nanopores, but mesopores are expected to exist on the transition from macropores to nanopores.

The nanopores start to grow at $3 \mathrm{~mA} / \mathrm{cm}^{2}$ and their growth of surface coverage is very minimal, while there are no well-etched grown macropores. Yet, its coverage is still minimal but little improved as the current increased to 4 and $5 \mathrm{~mA} / \mathrm{cm}^{2}$ (see Figures $5(\mathrm{a})-5(\mathrm{c})$ ). Thus, it seems that $\sim 3-5 \mathrm{~mA} / \mathrm{cm}^{2}$ is at a transition from macropores to nanopores regime. Here, it is worth noticing that the nanopores growth has unique orientation, somehow "island/hill" like and inhomogeneous growth over the surface as seen in Figures 5(a)-5(c), unlike that of macropores and electropolished surfaces [21, 22]. As the anodizing current increases, the growth of nanopores continues with improved surface coverage up to $15 \mathrm{~mA} / \mathrm{cm}^{2}$; however, the nanopores percentage of surface coverage reduces as the current density declines from 15 to $20 \mathrm{~mA} / \mathrm{cm}^{2}$. Compared to the transition from macropores to nanopores samples (samples anodized at $j=3-5 \mathrm{~mA} / \mathrm{cm}^{2}$ ), the nanopore dominating samples exposed a uniform distribution of the light under the UV light. Interestingly, nearly on all samples with nanopores craters are also observed, which are likely to be starting sites for the macropores. Thus, a mixture of nanopores and nuclei of macropores was present on all the samples. Three examples of the nanopores plus macropores nuclei are presented in Figures 4(a)-4(c).

Figure 6 shows the typical fast $I-V$ curves, in time, during the nanopore growth at $j=15 \mathrm{~mA} / \mathrm{cm}^{2}$. As shown in the figure, the fast $I-V$ gap tends to decline in time. A similar trend, reduction of $I-V$ gap in time, was also observed for the samples having other nanopores (e.g., nanopores at $j=$ $10 \mathrm{~mA} / \mathrm{cm}^{2}$ ). As observed in Figure 6, it is also evident that the gap in the first 50 min is significant and then suddenly diminishes just after about $75 \mathrm{~min}$ and remains relatively the same till $120 \mathrm{~min}$. This $I-V$ gap declining effect is more pronounced for the sample anodized at $j=15 \mathrm{~mA} / \mathrm{cm}^{2}$, which had the highest light intensity under the UV lamp. Here, it is important to note that though the nanopores have minimal coverage for $3-5 \mathrm{~mA} / \mathrm{cm}^{2}$, unlike the 10 or $15 \mathrm{~mA} / \mathrm{cm}^{2}$, a similar trend or reduction of the fast $I-V$ gap in time is observed.

The reduction in the fast $I-V$ gap, during nanopores formation, suggests that the amount of oxide formation/thickness decreases in time. Here, it is worth remembering that the formation of nanopores requires less oxide to form than macropores [23, 24]. Taking into account the fact that we have a transition range from macro- to nanoporous, we can assume that at the beginning of etching the macropores are favored (more oxides, bigger gap), and as the time goes the role is interchanged leading to less oxide, smaller gap. In short, the decrease of the $I-V$ gap is caused by the fact that as the time goes the nanopores start to dominate 


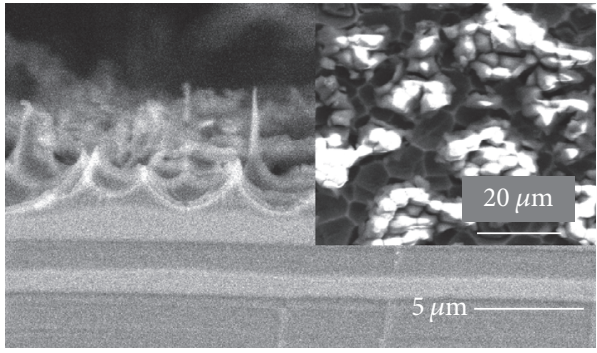

(a)

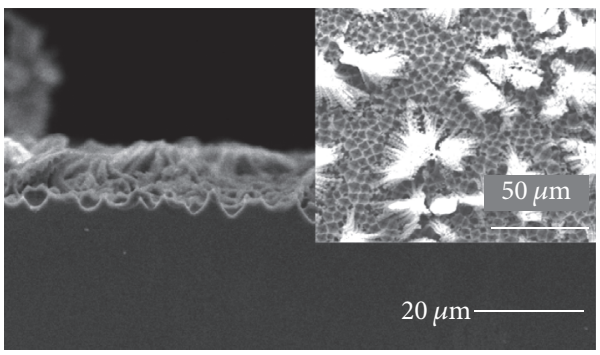

(c)

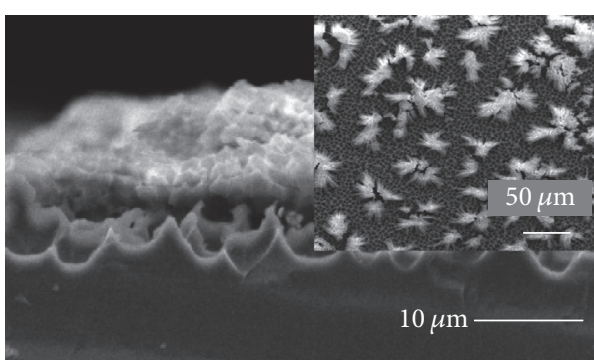

(b)

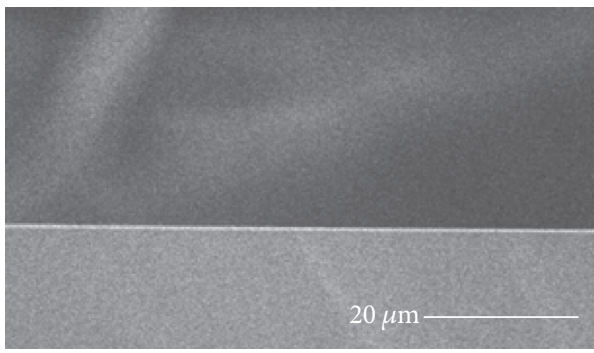

(d)

FIgURE 5: SEM images of the samples anodized for $120 \mathrm{~min}$ at $4 \mathrm{~mA} / \mathrm{cm}^{2}$ (a), $15 \mathrm{~mA} / \mathrm{cm}^{2}$ (b), $20 \mathrm{~mA} / \mathrm{cm}^{2}$ (c), and $25 \mathrm{~mA} / \mathrm{cm}^{2}$ (d). The first three images, with insets of surface view, show nanopores ((a), (b), and (c)), while the last (d) shows electropolishing.
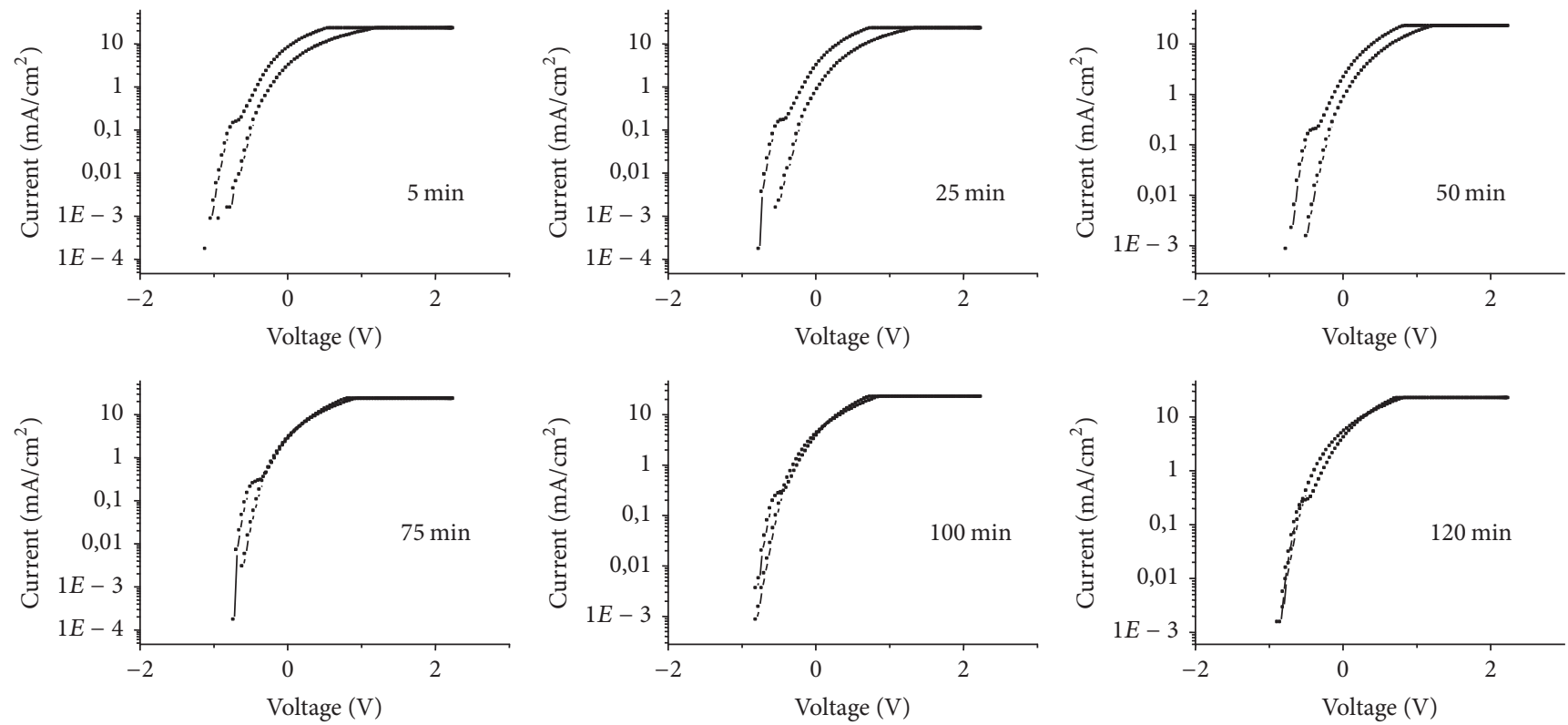

FIGURE 6: Typical in situ fast $I-V$ measurements (at 5, 25, 50, 75, 100, and 120 minutes) during the growth of nonporous structures at $15 \mathrm{~mA} / \mathrm{cm}^{2}$.

over the crater formation of macropores, thus leading to less oxide formation and consequently to a smaller fast $I-V$ gap.

3.3. Electropolishing. The transition from nanopores to electropolishing was observed for a current density value of $j=$ $20 \mathrm{~mA} / \mathrm{cm}^{2}$ (see Figure 5(c)). When the sample, anodized at $20 \mathrm{~mA} / \mathrm{cm}^{2}$, was investigated for photoluminescence property (under the UV lamp), it indicates the presence of nanopores. On the other hand, it should be noted that in this case a partial (minimal) surface of the sample was electropolished. Hence, it is a mediator of the previous/nanopores and the next/electropolishing regime.

An effective or complete electropolishing was obtained at $25 \mathrm{~mA} / \mathrm{cm}^{2}$ or higher (see Figure 5(d)). Figure 7 presents a typical in situ fast $I-V$ measurement during electropolishing. As expected, because of the electropolishing, the sample at $j=$ $25 \mathrm{~mA} / \mathrm{cm}^{2}$ shows a "high" $I-V$ gap and a relatively constant fast $I-V$ gap in time. This effect happens because of the fact 

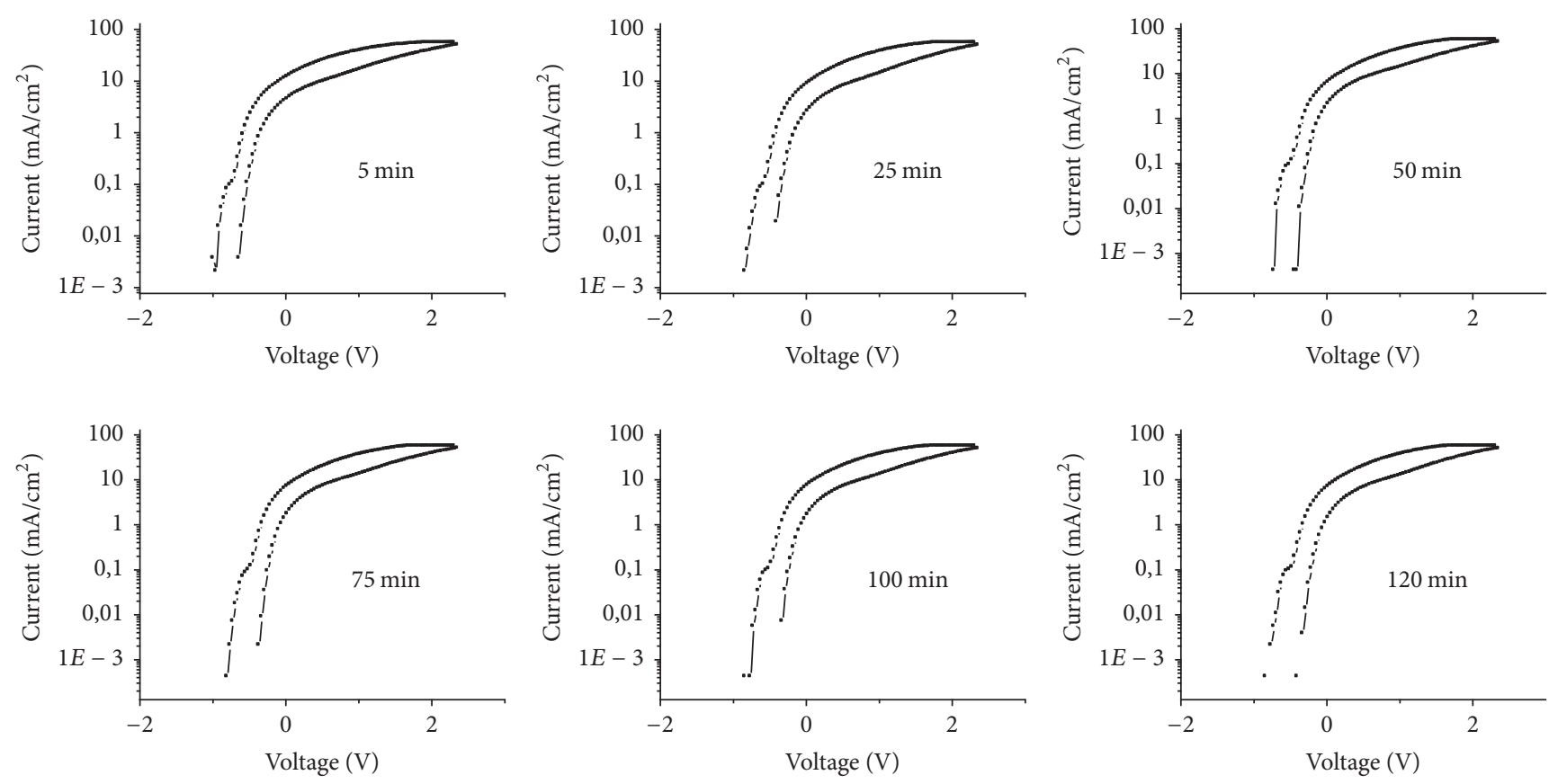

Figure 7: Typical in situ fast $I-V$ measurements during electropolishing at $25 \mathrm{~mA} / \mathrm{cm}^{2}$. The measurements were made at 5, 25, 50, 75, 100, and 120 minutes.

that the oxide forming molecules have almost equal concentrations at different times of the etching, because there are no deep pores which can create diffusion limitations. In other words, the oxidizing species at the silicon-etchant solution interface is not diffusion limited in time. Moreover, as seen in Figure 7, the $I-V$ gap (regardless of the time) is relatively high when compared to $I-V$ gap of the macro- or nanopores. Apparently, this effect is attributed to the relatively high rate of oxide formation in electropolishing than the pores formation $[2,25]$ and thus results in high $I-V$ gap.

The fast $I-V$ curves at $j=20 \mathrm{~mA} / \mathrm{cm}^{2}$ show a mediator phenomenon between 15 and $25 \mathrm{~mA} / \mathrm{cm}^{2}$ or mediator between nanopores and electropolishing. In other words, there is a reduction of the gap/area of the fast $I-V$ curves as a function of time, but it is not that much pronounced as in the previous case $\left(j=10\right.$ and $\left.15 \mathrm{~mA} / \mathrm{cm}^{2}\right)$ of the nanopores and it is not with constant gap over time, like the observed electropolishing case (see Figure 6). That is, the fast $I-V$ gap in time decreases slowly during a transition from nanopores to electropolishing and the reason remains the same as that of the other cases having nanopores, that is, having small creature like or macropores nuclei (more oxides, bigger gap) to nanopores (less oxide, smaller gap) and partially minimal electropolishing.

\section{Conclusion}

A new method called in situ fast $I-V$ curve method is used to study the growth of porous structures on p-type Si. The method is used to study the relative rate of oxide formation at different pore depth or time, while pore etching and electropolishing is proceeding. The macropores grown in this study are not significantly deep (up to $14 \mu \mathrm{m}$ ) and more importantly they are not straight, instead the pores exhibit spongy like, cavity, or tumbler like structure. The rate of oxide formation is determined by the diffusion rate of oxide forming molecules which in turn depends on the pore morphology. As a result, the in situ fast $I-V$ gap for macropores increases in time, which is attributed to the pore morphology favoring the diffusion rate of oxide forming or water molecules. On the other hand, in nanopores regime, the $I-V$ gap decreases in time. This is because at the beginning of etching the macropores are favored, more oxides with bigger gap, while as the time goes nanopores overtook, less oxides with smaller gap. Furthermore, the electropolishing process (at $25 \mathrm{~mA} / \mathrm{cm}^{2}$ ) resulted in two major features: constant gap of the fast $I-V$ in time and relatively high $I-V$ gaps compared to the micro/nanopores regimes. The effect is attributed to relatively high rate of oxide formation of electropolishing than the pores formation (high $I-V$ gap) and the absence of diffusion limitation unlike that of pore formation (relatively no $I-V$ gap change in time). The fast $I-V$ method has a potential for its development into a more advanced study, to investigate the rate of oxidation, either by changing different parameters of the electrochemical etching conditions or extending its application in other semiconductor materials etched electrochemically.

\section{Conflicts of Interest}

The author declares that they have no conflicts of interest.

\section{Acknowledgments}

The author acknowledges Professor H. Föll in the Institute of Materials Science, Faculty of Engineering, at ChristianAlbrechts-University of Kiel for his lab facility and scientific 
discussions during the study. The author is also grateful to Dr. S. Langa, Dr. J. Carstensen, and Professor G. Tsidu for their fruitful discussion and kind technical assistance during the study. Besides, grant support from the International Science Program (ISP, 2016) in relation to publishing the experimental work, for Physics Department at Addis Ababa University (AAU), is greatly acknowledged.

\section{References}

[1] K. Seeger, Semiconductor Physics: An Introduction, Springer, Berlin, Germany, 1985.

[2] H. Föll, M. Christophersen, J. Carstensen, and G. Hasse, "Formation and application of porous silicon," Materials Science and Engineering R, vol. 39, no. 4, pp. 93-141, 2002.

[3] L. Canham, Ed., Properties of Porous Silicon, Institution of Electrical Engineers, London, UK, 1997.

[4] V. Lehmann and H. Föll, "Formation mechanism and properties of electrochemically etched trenches in n-type silicon," Journal of the Electrochemical Society, vol. 137, no. 2, pp. 653-659, 1990.

[5] C. Pacholski, "Photonic crystal sensors based on porous silicon," Sensors, vol. 13, no. 4, pp. 4694-4713, 2013.

[6] C. Jamois, R. B. Wehrspohn, L. C. Andreani, C. Hermann, O. Hess, and U. Gösele, "Silicon-based two-dimensional photonic crystal waveguides," Photonics and NanostructuresFundamentals and Applications, vol. 1, no. 1, pp. 1-13, 2003.

[7] A. Tiwari and B. Raj, Eds., Materials and Failures in MEMS and NEMS, John Wiley \& Sons, VCH, Weinheim, Germany, 2015.

[8] K. A. A. Min-Dianey, H.-C. Zhang, N.-L. M'Bouana, C. Su, and X. Xia, "Modeling of spectral energy density as thermal radiation characteristic on the basis of porous silicon photonic crystals," Computational Materials Science, vol. 136, pp. 306-314, 2017.

[9] D. Losic and A. Santos, Eds., Electrochemically Engineered Nanoporous Materials: Methods, Properties and Applications, Springer, New York, NY, USA, 2015.

[10] H. Gerischer and C. V. Tobias, Eds., Advances in Electrochemical Science and Engineering, vol. 4, John Wiley \& Sons, VCH, Weinheim, Germany, 2008.

[11] J. Crank, The Mathematics of Diffusion, Clarendon Press, Oxford, UK, 1975.

[12] H. Foll, J. Carstensen, M. Christophersen, and G. Hasse, "A new view of silicon electrochemistry," Physica Status Solidi (A), vol. 182, no. 1, pp. 7-16, 2000.

[13] A. Benor, In situ measurements during the growth of porous structures on p-type [Master Thesis], University of Kiel, Kiel, Germany, 2003.

[14] K. J. Chao, S. C. Kao, C. M. Yang, M. S. Hseu, and T. G. Tsai, "Formation of high aspect ratio macropore array on p-type silicon," Electrochemical and Solid-State Letters, vol. 3, no. 10, pp. 489-492, 2000.

[15] G. X. Zhang, Modern Aspects of Electrochemistry, vol. 39, Springer, New York, NY, USA, 2005.

[16] M. Zhao, A. McCormack, and M. Keswani, "The formation mechanism of gradient porous $\mathrm{Si}$ in a contactless electrochemical process," Journal of Materials Chemistry C, vol. 4, no. 19, pp. 4204-4210, 2016.

[17] M. Galinsky, U. Sénéchal, and C. Breitkopf, "The impact of microstructure geometry on the mass transport in artificial pores: a numerical approach," Modelling and Simulation in Engineering, vol. 2014, Article ID 109036, 7 pages, 2014.
[18] M. N. Khan, M. A. M. Khan, A. S. Al Dwayyan, and J. P. Labis, "Comparative study on electronic, emission, spontaneous property of porous silicon in different solvents," Journal of Nanomaterials, vol. 2014, Article ID 682571, 14 pages, 2014.

[19] S. Li, X. Chen, W. Ma et al., "An innovative metal ions sensitive "test paper" based on virgin nanoporous silicon wafer: highly selective to copper(II)," Scientific Reports, vol. 6, article 36654, 2016.

[20] J. A. Roger, M. G. Blanchin, B. Canut, V. S. Teodorescu, S. Letant, and J. C. Vial, "Tin oxide growth in nanoporous silicon: an approach to an efficient solid state electrode," Semiconductor Science and Technology, vol. 14, no. 11, pp. L29-L32, 1999.

[21] G. Korotcenkov and B. K. Cho, "Silicon porosification: state of the art," Critical Reviews in Solid State and Materials Sciences, vol. 35, no. 3, pp. 153-260, 2010.

[22] N. Winter, M. Becton, L. Zhang, and X. Wang, "Effects of pore design on mechanical properties of nanoporous silicon," Acta Materialia, vol. 124, pp. 127-136, 2017.

[23] E. K. Propst and P. A. Kohl, "Electrochemical oxidation of silicon and formation of porous silicon in acetonitrile," Journal of the Electrochemical Society, vol. 141, no. 4, pp. 1006-1013, 1994.

[24] P. Granitzer and K. Rumpf, "Porous silicon-a versatile host material," Materials, vol. 3, no. 2, pp. 943-998, 2010.

[25] M. J. Sailor, Porous Silicon in Practice: Preparation, Characterization and Applications, John Wiley \& Sons, Weinheim, Germany, 2012. 

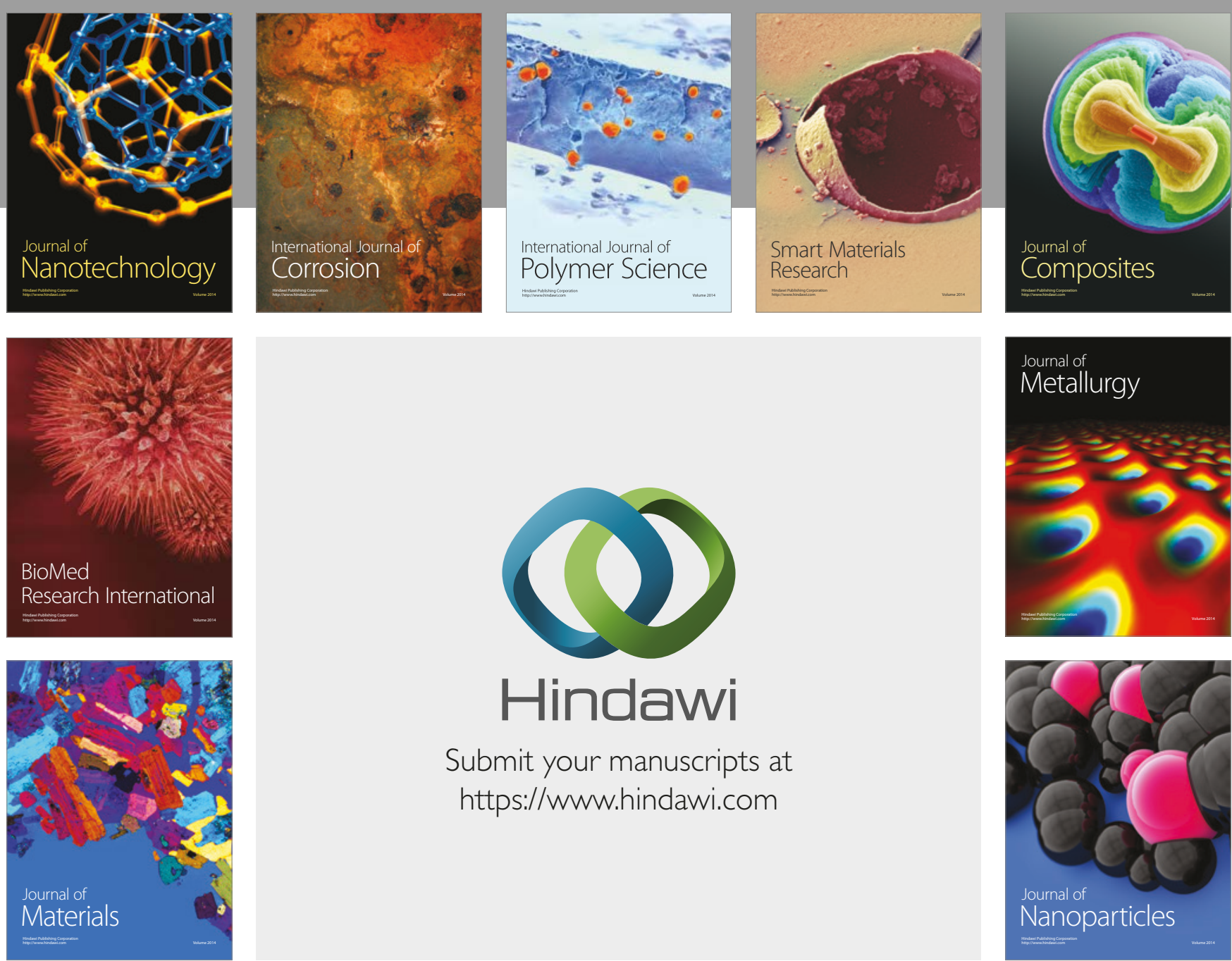

\section{Hindawi}

Submit your manuscripts at

https://www.hindawi.com
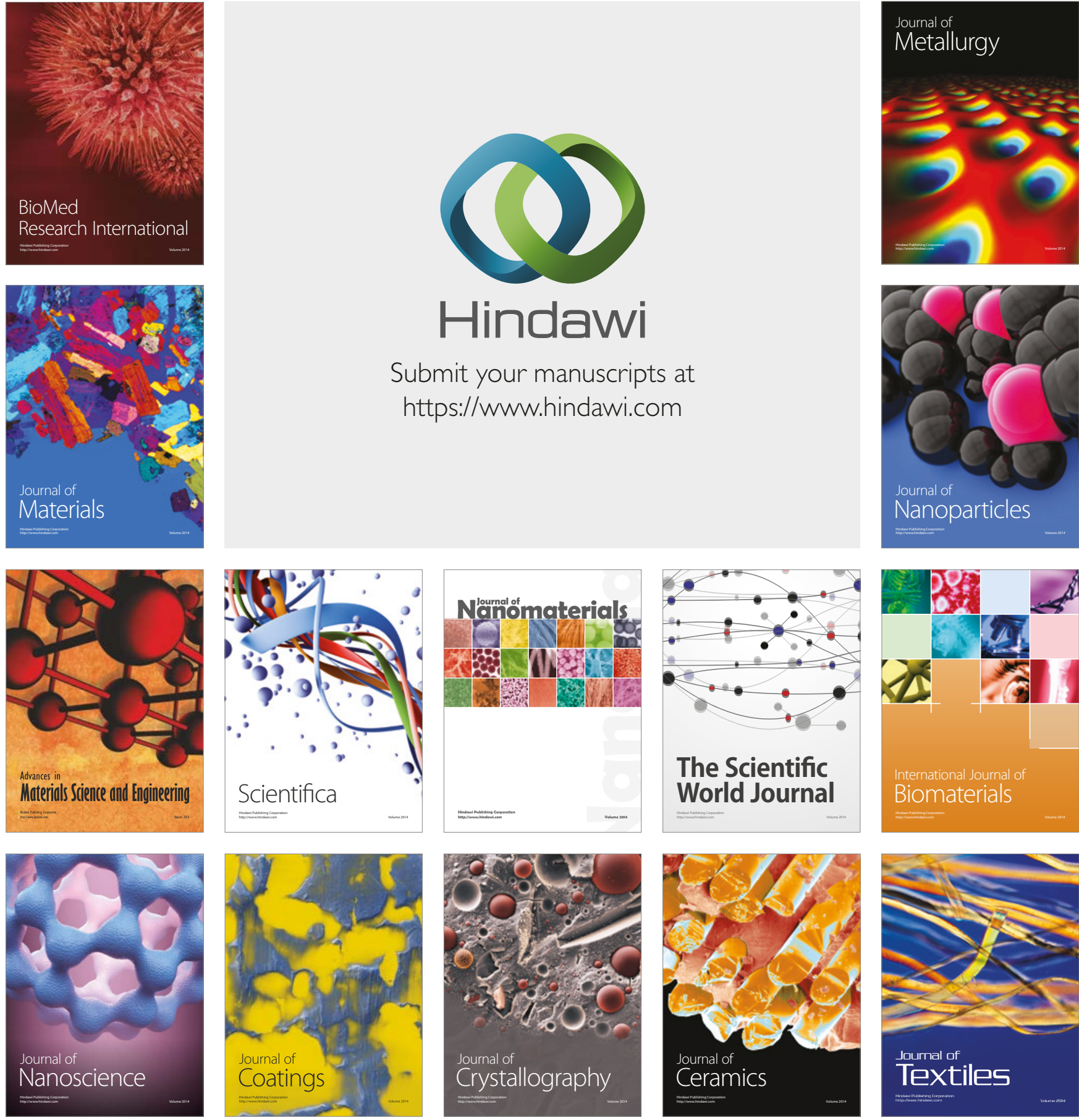

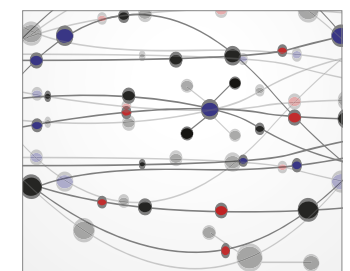

The Scientific World Journal
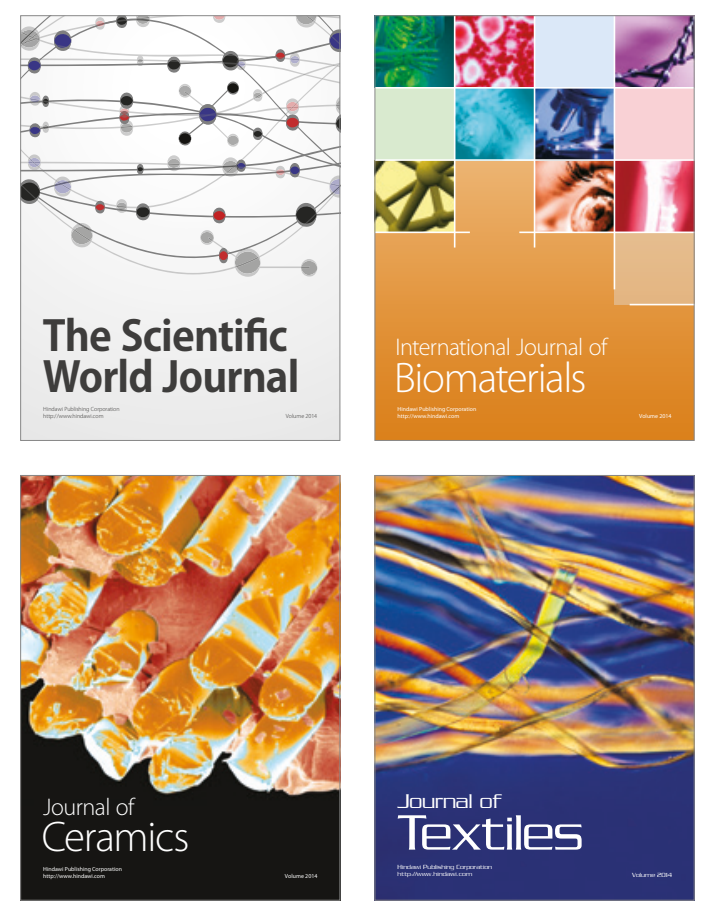